\title{
Comment to "Magnetic resonance imaging (MRI) findings of bilateral thalamic involvement in severe paroxysmal sympathetic hyperactivity (PSH): a pediatric case series" by Mrkobrada, et al
}

\author{
Dhaval P. Shukla ${ }^{1}$ (D) \\ Received: 24 November 2015 / Accepted: 4 December 2015 / Published online: 11 December 2015 \\ (C) Springer-Verlag Berlin Heidelberg 2015
}

\section{Dear Editor:}

We read with great interest article titled "Magnetic resonance imaging (MRI) findings of bilateral thalamic involvement in severe paroxysmal sympathetic hyperactivity (PSH): a pediatric case series" by Mrkobrada, et al. [4]. The authors conclude that damage to bilateral thalami may be required for symptom development in the pathophysiology of severe (PSH), based on their experience of three cases in children. In their series besides thalami, cortex, subcortical white matter, caudate and putamen were also involved.

The central autonomic network is distributed in the entire neuraxis, and widespread disruption of this network leads to PSH. The neuroanatomical basis cannot be ascertained, and involvement of thalami cannot be categorically stated in the pathophysiology of PSH. We have reported PSH in children with severe traumatic brain injury and moyamoya disease $[1,2]$. The MRI in children with TBI revealed diffuse axonal injury, indicating widespread disruption of central autonomic network. In the child with moyamoya disease,

Dhaval P. Shukla

neurodhaval@rediffmail.com

1 National Institute of Mental Health and Neuro Sciences, Bangalore, Karnataka, India imaging finding was of bilateral middle cerebral artery ischemia involving insula, which is a part of central autonomic network.

In a diffusion tensor imaging study of patients with PSH, decreased fractional anisotropy in the splenium of the corpus callosum and in the posterior limb of the internal capsule discriminated PSH vs no PSH with excellent accuracy [3]. There is a need of more studies to conclude the anatomical basis of PSH in brain injury.

\section{References:}

1. Deepika A, Reddy M, Shukla D (2014) Paroxysmal sympathetic hyperactivity in a child with moyamoya disease. J Neurosurg Anesthesiol 26:87-88

2. Deepika A, Mathew MJ, Kumar SA, Devi BI, Shukla D (2015) Paroxysmal sympathetic hyperactivity in pediatric traumatic brain injury: a case series of four patients. Auton Neurosci. Doi.10.1016/ j.autneu.2015.08.003.

3. Hinson HE, Puybasset L, Weiss N, Perlbarg V, Benali H, Galanaud D, Lasarev M, Stevens RD (2015) Neuro imaging for coma emergence, recovery (NICER) consortium. Neuroanatomical basis of paroxysmal sympathetic hyperactivity: a diffusion tensor imaging analysis Brain Inj 29:455-61. Doi:10. 3109/02699052.2014.995229.

4. Mrkobrada S, Wei XC, Gnanakumar V (2015) Magnetic resonance imaging findings of bilateral thalamic involvement in severe paroxysmal sympathetic hyperactivity: a pediatric case series. Childs Nerv Syst. Doi: 10.1007/s00381-015-2931-z. 